\title{
Fichte und Goethe.
}

\author{
Von Ernst Bergmann.
}

"Wir haben in einer Zeit gelebt und "die Nachwelt wird uns als Zeitgenossen zu Nachbarn machen; aber wie wenig haben wir uns vereinigt."

schiller.

Die beiden grössten Deutschen haben Fichtes Leben gestaltet. Kant entschied sein inneres, Goethe sein äusseres Schicksal. Er berief inn nach Jena und verstiess inn wieder.

Warum hat Goethe, der Allmächtige, den Sieg der Obskuranten sanktioniert? Als die Waimarer Regierung schwankte, votierte er gegen Fichte. Die Frage ist tiefer zu fassen. Wie hat sich Fichtes Persönlichkeit im Geist des Weltumfassenden gespiegelt? Das Urteil Goethes gleicht dem einer absoluten Menschheit. Wie Goethe ihn sah, so war Fichte.

Das Verhältnis zu Schiller ist weniger problematisch. Wechselseitiges Wohlwollen auf Achtung gegründet, auch Freundschaft, innere Wärme mehr von Seiten Fichtes. Ein Missverständnis trübt zeitweilig das gute Einvernehmen beider, nicht obne tiefere Spuren zu hinterlassen. Wenigstens beweist Schiller nach Fichtes Entlassung wenig freundschaftliche Gesinnung gegen ihn (an Goethe, 14. Juni 1799). Die beiden Gattinnen unterhalten einen regen Verkehr. Das letzte Trennende ist die Spekulation, trotz der Gemeinsamkeit der idealistischen Weltbetrachtung, trotz des beiderseitigen Kantianertums. In dem Augenblick, da Fichte Philosoph wird, wird Schiller wieder Dichter. Er hat in Metaphern gedacht, mit der Einbildungskraft raisonniert. An Fichtes systematischer Methodik erkannte er vielleicht das inuere Unwabre seiner $\mathrm{Be}$ mühungen. Er wendet sich unbefriedigt ab von der Spekulation, vom Logos zurück zum Bios. Der Dichter ist der einzig wahre Mensch. Der beste Philosoph ist nur eine Karikatur gegen ihn.

Ganz anders Goethe. Er schwankt nicht zwischen den beiden Extremen. Sein vermittelnder Geist denkt auf den Einklang beider. Kantatudien XX. 
In diesem Sinne bedeutet er Fichte beim Empfang der ersten Bogen der Wissenschaftslehre. Durch die wissenschaftliche Begründung dessen, „worüber die Natur mit sich selbst in der Stille schon lange einig zu sein scheint", werden Sie dem menschlichen Geschlecht eine unschätzbare Wohltat erweisen und werden sich um jeden Denkenden und Fühlenden verdient machen (24. Juni 1794). Goethe ist Pragmatist. Die Spékulation diene der Wirklichkeit. In Gesprächen führt er Fichte auf diese Einsicht. Fichte nimmt sie an. Sie klingt wieder aus Briefen an Jacobi und Reinhold (Leben, I, $178 \mathrm{ff}$.). Sie wird zum Hauptgedanken des „Sonnenklaren Berichts". "Nichts hat unbedingten Wert und Bedeutung als das Leben; alles übrige Denken, Dichten, Wissen hat nur Wert, insofern es auf irgend eine Weise sich auf das Leben bezieht, von inm ausgeht und in dasselbe zurückzulaufen beabsichtigt. Dies ist die Tendenz meiner Philosophie" (333). Hier wird Fichte zum Schüler Goethes, freilich etwas spät. An diesem Normalmenschen gewahrt er das Streben zum Ausgleich zwischen Leben und Denken. Ich (Goethe) werde İhnen den grössten Dank schuldig sein, „wenn Sie mich endlich mit den Philosophen versöhnen, die ich nie entbehren und mit denen ich mich niemals vereinigen konnte. (24. Juni 1794).

Genauer betrachtet: Goethes Abneignng gegen die Spekulation ist gross. Begriffliche Abstraktion ist seiner konkretisierenden Geistesanlage fremder noch als Schiller. An sich muss die Spekulation dem geborenen Künstler als eine "falsche Tendenz" erscheinen (an Mayer, 18. März 1797). Nụr kann man ihr nicht ausweichen. Alles spekuliert rings unher, in Weimar und Jena. Und Goethe ist wissenschaftlich fortwährend interessiert. Sein umhersuchender Geist greift nach allem, was nur irgend Belehrung verspricht. Sein Universalismus verlangt nach der Fülle der Erscheinungen, nach jedem Abglanz menschlichen Seins im Spiegel des Ewigen. Also! Man muss schon ein wenig hinabsehen in den philosophischen Kampfplatz, "will man mit der Zeit gehen" (an Schiller, 25. Nov. 1795). So zwingt er sich zu Fichte, wie er sich zu Kant gezwungen. Der Gedanke, dass eine höchste Lèhre von aller Wissenschaft aus apriorischen Sätzen deduziert werden soll, reizt seine Erwartung. Noch mehr vielleicht die neue Lebensform Fichte, die Art des Unendlichen, in Fichte zu sein. Hat sich doch Fichte bereits "mit Grossheit" über menschliche Dinge ausgesprochen. So ist Goethe sein warmer Freund schon vor der persönlichen Bekanntschaft 
(Bertuch an Fichte, Leben I, 196). Wie angenehm, wie interessant, diesen Geist in erreichbarer Nähe zu wissen (Leben, I, 266). Wio viel wird sich da besprechen lassen, Neues erlernen, Altes befestigen oder berichtigen. Goethe ist es gewesen, der zu einer Zeit, da Reinhold noch schwankte, am 27. Juli 1793, die Berufung Fichtes bereits erwog und anregte (an Voigt).

Die Enttäuschung konnte bei diesen hochgespannten Erwartungen nicht ausbleiben. Sie kommt von zwei Seiten, von der Persönlichkeit und vom Systen.

Anfangs freilich herrscht eitel Harmonie. Goethe empfängt den Philosophen (am 20. Mai 1794) „höchst freundschaftlich" (Fichte an Johanna, I, 209) und bewährt sich auch fernerhin als sein "warmer Freund" (I, 215, 216, 262). Bis zum Herbst des Jahres stehen beide Männer im Briefwechsel. ${ }^{1}$ ) Fichte findet Goethe weit empfänglicher für Spekulation als Schiller (I, 108). Gegen Humboldt äussert er, "dass er Goethe für die Spekulation zu gewinnen wünschte." 2) Goethe habe ihm sein (Fichtes) System so klar und bündig dargelegt, dass er es selbst nicht klarer vermocht hätte (?): Auch Herbart weiss, dass Goethe Fichten am besten verstanden habe. ${ }^{3}$ ) Noch 1797 geht Goethe in Jena abends mit Fichte die "Neue Darstellung der Wissenschaftslehre " durch (an Mayer, 18. März 1797). Er glaubt selbst, Fichten zu verstehen (an Fichte, 24. Juni 1794). Dies war wohl nie ganz der Fall, trotz ausführlicher Auszüge aus der Wissenschaftslehre, die noch vorhanden sind:

Ja, Fichte schreibt anfangs die Wissenschaftslehre eigentlich n ur für Goethe. Er wende sich mit ihr an „die reinste Geistigkeit des Gefühls", wie sie Goethe repräsentiere. ") Goethes Gefühl soll den Probierstein dieser Philosophie abgeben. Kann ich mir Goethen als völlig damit zufrieden denken, so fragt er sich bei der Ausarbeitung. ${ }^{5}$ ) Fichtes Verehrung für Goethe kennt keine Grenzen. Tasso und Iphigenie bewundert er schon früh (Leben, I, 114). Später erklärt er die "Natürliche Tochter", die er in Berlin zweimal (ausgepfiffen') sah, für das „dermalige höchste Meisterstück des Meisters. " ${ }^{\circ}$ )

1) Goethe-Jahrbuch, Bd. 15 (1894).

2) Briefwechsel $z$ wischen Schiller und Humboldt, S. $108 \mathrm{f}$.

?) 28. Aug. 1795. Allihns Zeitschrift f. exakte Philos. 1860, S. 323.

4) An Goethe, 21. Juni 1794. Goethe-Jahrbuch, XV.

5) An Goethe, 30. Sept. 1794. Ebenda.

f An Schiller, 20. Juli 1803. Leben, II, 397. 
Und Goethes Eindruck? Eine Persönlichkeit, ganz zweifellos. Einer der vorzüglichsten Köpfe und - ,je älter man wird, je mehr schätzt man Naturgaben, weil sie durch nichts können angeschafft werden ${ }^{\mu}$ (an Schlosser, 30. Aug. 1799). Schon dies ein bedingtes Lob. Der Diamant ist roh, unabgeschliffen. Aber sonst: viel Behendigkeit des Geistes, ein rigider Sinn, ein Mensch für Gespräch und Mitteilung, der sich gern in alles einlässt, wie Goethe das liebte (an Jacobi, 2. Febr. 1795). „Es konversiert sich auch mit ihm sehr gut" (an die Kalb, 28. Juni 1794). Von Reinhold galt das nicht. Er war verschlossen. Dies die ersten Impressionen. Goethe vermittelt die Verbindung mit Jacobi, um dessen Verständnis Fichte lebenslänglich gerungen.

Und dann gar bald: "ein wunderlicher Kauz", sonderbar wie sein System, das Goethe in Wahrheit nie verstanden (an Jacobi, 2. Febr. 1795). War es der schon damals offen zur Schau getragene Demokratismus des Leinewebersohnes, der den monarchischkonservativ gesinnten Weimarer Aristokraten und Staatsminister abstiess, jene Sanskulottentournüre, deren. Nichtvorhandensein ihm an dem massvolleren Schelling so angenehm auffiel (an Voigt, 29. Mai 1798)? Sicherlich nicht allein.

Der Grund des mangelnden Einverständnisses zwischen den beiden grossen Deutschen liegt tíefer, ist allerpersönlichster Natur. Goethes vielgerühmte Objektivität gegen fremde Lebensformen hatte ihre ganz bestimmten Grenzen. Schiller hatte es erfahren, vor 1794, und mancher andere. Fichte erfuhr es jetzt. Fichte stand $1794 \mathrm{da}$, wo Goethe vor 20 Jahren gestanden, Schiller vor 10, mitten in brausenden Sturm und Drang. Goethe aber kam aus Griechenland. Soeben hatte er sich den Olympischen Stuhl zurechtgeruickt, um hinfort an goldenen Tischen das wunderbare Fest des Lebens zu feiern. Da erhebt sich in seiner allernächsten Nähe das Haupt des emporbrechenden Titanen. Das "unheimlich Imponierende", Inkommensurable an Fichte soll Goethe geschreckt haben. Mag sein. Eine Zeitlang sah der gesetzte Mann nur den unruhig hin- und herfahrenden Kopf in der Nachbarschaft, der mit seinen fortwährenden Händeln die Behörden belästigte, den geordneten Gang der ministeriellen Geschäfte unterbrach; den betriebsamen Reformer mit halbausgegorenen Ideen, die das abgeklärte hellenische Weltbild verwirrten, das man sich im Kreise derer um Humboldt zurechtgelegt. Sicher ist, dass Goethe im Betragen des Lausitzer Bauernsohnes „etwas Fratzen- 
haftes" gewahrte (an Voigt, 12. März 1800), eine Disharmonie, ein Stück Karikatur wie Anselm Feuerbach, ${ }^{1}$ ) Mangel an Delikatesse, Feinheit, Humanität wie Forberg, einen urorganischen Zug. Etwas Unapollinisches, vielleicht Bacchantisch-Wirres in und an Fichte, sein lautes, stürmendes Wesen in jener Zeit, seine gewaltsame Art zu wirken, die Schroffheit, Rücksichtslosigkeit seiner Energie, sein trotziges, forderndes, polterndes Kraftmenschentum, dieses Schlagen auf die Tischkante, dieser gereizte Ton in seinen Briefen, diese Masslosigkeit, dieser Mangel an Selbstzucht und an der "eigentlichen Lebensweisheit", die immer erst "von oben" in alle Reformunternehmungen Fichtes hineingebracht werden musste, kurz all das, was einem Winckelmannischen Auge als unerhörte Verzerrung edler, grosser Linien erscheinen musste, das ist es, was dem Iphigeniadichter auf die Dauer unerträglich wird. Ich bin politisch verdächtigt. Will nich der Fürst schützen? Ia oder nein? Man heisst mich Sabbathschänder. Ich verlange ein Gesetz. Den Senat beuge ich mich nicht. Und nach Mittage kann ich nicht lesen. Ich will den offenen Geist meiner Hörer in den Morgenstunden, nicht ihren gefüllten Bauch, der keine Ohren hat (an Goethe, 29. Nov. 1794). Gleich darauf der Handel mit den Orden, der Atheismusstreit. Genug, genug! Möchte doch endlieh das unaugenehme Geräusch aus der Nachbarschaft verschwinden. "Goethe kann nicht gerecht gegen Sie sein", schreibt Schiller an Fichte, als er schon im Osmanustädter Exil weilte (3. Aug. 1795). „Sonderbar genug ist es, dass Sie von mir erst hören müssen, wie wenig Goethe dazu taugt, Ihre Partei zu ergreifen." Es handelte sich um den Horenaufsatz. Fichte hatte an Goethe appelliert.

Die Lebensformen beider Männer weichen in jenen Jahren grundsätzlich auseinauder und nähern sich einander erst später, wenn Fichte seine lärmende Autonomie gezügelt hat. Damals aber ergreift Goethe die erste passende Gelegenheit, da Fichte eine Ungeschicklichkeit begangen, um den unbequemen Nachbar durch eine Rechtsverletzung aus Jena zu entfernen. Zugleich beklagt er den unersetzlichen Verlust. Gab es keinen anderen Ausweg? Bald darauf besucht inn Steffens. Er gewahrt „einige Verlegenheit" in Goethes Benehmen. ${ }^{2}$ ) Steffens war damals eifrig für Fichte tätig. Goethe wusste das.

3) Leben und Wirken A. v. Feuerbachs. 1851. I, $51 \mathrm{f}$.

2) Was ich erlebte. IV, 166. 
Kommt hinzu die Enttäuschung über die Theorie. Schon im April 1794 ahnt Goethe, Fichte werde "in ein sonderbares Horn“ stossen. Auch hier ein Fratzenhaftes, in jenen „äusserst zugespitzten Apices“ (Kant) der Jenenser Wissenschaftslehre, die einem natürlichen Wabrheitssinn als stechend erscheinen mussten. Fichtes Sittenlehre, seine Bestimmung des Menschen scheint Goethe gar nicht zu kennen. Es ist in Goethes Briefen eigentlich immer nur vom theoretischen Teil der Wissenschaftslehre die Rede, nirgends von der ethisch-pädagogischen Bedeutnng des Idealismus überhaupt. Hier aber hätte er den Weg zum apostolischen Fichte und den bei ihm früh hervortretenden grossen Humanitätsideen finden müssen, die dann am Schluss des zweiten Faust aufleuchten. „Solch ein Gewimmel möcht ich sehn!" Klingt es nicht wie ein Traum aus . Fichte, wie ein Wissen um ferne verklärte Mensehheitsalter, wie die Hoffnung auf das baldige Kommen des Vernunitand Freiheitsreiches auf Erden! „Auf freiem Grund mit freiem Volk zu stehn!“ - Aber Goethe sieht in Fichte nirgends den grossen Ethiker und Weltheiland, den Staats- und Geschichtsphilosophen, immer nur den spintisierenden Theoretiker, ein Schulbeispiel für das berühmte Tier auf dürrer Heide. Im Sommer 1795 heisst Fichte in Goethes Briefen ständig "das Osmannstädter Ich“ (an Schiller, Juli, 1795). Wie flach weiss er gegen Voigt zu witzeln über die "unhöflichen Nichtichs" in Fichtes Fensterscheiben (10. April 1795)! Auf die groteske Ichidee der frühesten, noch unausgereiften Fichteschen Versuche starrt er fasziniert. Sie beschäftigt ihn unablässig. In der Walpurgisnacht lässt er den Systematiker des unbewusst produzierenden Ich angesichts der Fülle unreinlicher Gesichte des Hexensabbaths das seufzend-ironische Geständnis ablegen:

Die Phantasie in meinem Sinn

Ist diesmal gar zu herrisch!

Fürwabr, wenn ich das alles bin,

Dann bin ich heute närrisch.

Und doch ist es gerade die Idee des produktiven Ich, die zündend in Goethes Dichterwelt eingeschlagen. Sein erkenntniskritisch nur wenig geschulter Geist deutet diese Idee grobanschaulich im Sinne eines die Welt buchstäblich erschaffenden Ich. Und ob er diese naive Deutung des Pseudo-Fichteaners sogleich selbst mephistophelisch-erhaben ironisiert - Original, fabr hin in deiner Pracht! -, sein künstlerisches Genie verwebt sich doch mit In- 
brunst in die verführerischen Möglichkeiten einer solchen Voraussetzung, die körperliche Welt entquölle unserm „innerlichen Licht".

Die Welt, sie war nicht, eh ich sie erschuf;

Die Sonne führt ich aus dem Meer herauf;

Mit mir begann der Mond des Wechsels Lauf;

Da schmückte sich der Tag auf meinen Wegen,

Die Erde grünte, blühte mir entgegen;

Auf meinen: Wink, in jener ersten Nacht,

Entfaltete sich aller Sterne Pracht. usw.

Kommt hinzu Goethes "entschiedener Hass" gegen Anmassung jeder Art. ${ }^{1}$ ) Man gibt mir Ratschläge, die ich sicher befolgen würde, wenn ich „Parmenio wäre." So Fichte wörtlich an Goethe (24. Juni 1794). ${ }^{2}$ ) Also ein Alexander! Und dann: Wissen Sie, ich kann sehr wohl einen grossen Mann (Karl August) verehren, selbst wenn er ein Fürst ist. Und weiter, jene Intoleranz des Wissenschaftslehrers! Was sind sie alle, die Platone und Leibnize -, servum dogmaticorum pecus! Und Kant hat geirrt, ein Dreiviertelskopf. Fichte ist der erste Philosoph. Als wenn überhaupt einer allein die Wahrheit ergreifen könnte! „Nur sämtliche Menschen erkennen die Natur, nur sämtliche Menschen leben das Menschliche", schreibt Goethe an Schiller (8. Mai 1798) mit Bezugnahme auf Fichtes Überhebung.

Kurz vor Beginn des Atheismusstreites steht es für Goethe fest: "An eine engere Verbindung mit ihm ist nicht zu denken" (an Schiller, 29. Aug. 1798). Interessant wird es immer sein, inn in der Nähe zu haben. Schiller mag sich in Jena mit ihm berühren und davon Nutzen ziehen und auch Fichten das Verbältnis heilsam werden lassen. Aber innerhalb der Goetheschen Lebensharmonie bedeutet die Seinsform dieses grobschlächtigen Ungriechen einen fremden Akkord. Erlaubt er sich gar die Sprache einer "nnverschämten Präoccupation" gegen ein Gouvernement, so ist es mit der Geduld des Herrn Geheimrat zu Ende. Er würde gegen seinen eigenen Sohn votieren, wenn er "gewisse Formen" vernachlässigte. ${ }^{3}$ )

Um die Katastrophe vollkommen za machen, erklärt sich um die gleiche Zeit auch Kant gegen die Wissenschaftslehre. Nun also, so weiss man denn endlich, was an ihr ist. Und Goethe nimmt von diesem Bannstrahl eines Halbtoten, der nach Schelling die „Selbst-

1) An Jacobi mit Bezug auf Fichte, 2. Jan. 1800.

2) Goethe-Jahrbuch, 15. Bd.

3) An Schiller, 23. Sept. 1803. 
annihili erung" Kants bedeutete, befriedigt Notiz (an Fichte, 12. Sept 1799). Ein Schwanken, ein Zweifel ist nun nicht mehr möglich. Á̉vòs है $\varphi \alpha$. Man hat also recht gehandelt, dass man keine Milde walten liess. „Diese Herren kauen sämtlich ihren eigenen Narren beständig wieder, ruminieren ihr Ich." Aus dieser Schule ist wenig Freude und Nutzen zu erhoffen So klingt es nach Fichtes Weggang hart aus Goethes Munde, und zwar bezeichnenderweise an W. v. Humboldt (30. Aug. 1799). Und doch wird ihm das eine Auge feucht. Er fürchtet Fichten "für sich und die Welt verloren" (an Schlosser, 30. Aug. 1799). „Seine jetzige Lage muss ihm zu seinen übrigen Fratzen noch Bitterkeit hinzufügen." Warum hat Goethe keinen Finger gerührt, um die Existenz, die er vernichtet, an einem andern Ort wieder aufzubaun? Wie gut hätte dieser Zug in das Bild des grossen Mannes gepasst! Statt dessen verhinderte man Fichtes Ansiedlung in Rudolstadt. ${ }^{1}$ ) Man wollte ihn gănzlich aus der Nachbarschaft entfernen. Welch bittrer Ton in Fichtes Brief an Reinhold vom 22. Mai 1799! Schon sieht er sich als einen zweiten Rousseau umherirren. „Ich glaube fest, dass ich ohne den besonderen Schutz eines Fürsten nirgends auf deutschem Boden sicher bin:"

Wie er nun hinaus ist, wie nun die ewige Belästigung der Behörden ein Ende hat, mildert sich die Denkweise und in das Streben, gerecht zu sein, mischt sich ein Ton von Selbstanklage. Schmerzlich, dass wir ihn verlieren mussten, „dass seine thörichte Anmassung inn aus einer Existenz warf, die er auf dem weiten Erdenrund nicht wiederfinden wird“. Er war doch ein Klotz Natur. Dergleichen lerñt man schätzen. Mehr noch, er war eine "von Hause aus vornehme Natur, die an sich selbst glaubt und also auch an das Beste glauben muss, dessen der Mensch auf seiner höchșten Stufe sich fähig halten darf" (an Jacobi, 2. Jan. 1800). Ein solcher Anblick ist immer wohltätig. Denkt Goethe an Fichten, wenn er sich im gleichen Brief ungerecht schilt „gegen das wahre ideale Gute im Menschen, das sich in der Erfahrung nicht wohl ganz rein zeigen kann?" Wunderbar tiefe Einsicht! Die Zeit (oder Jacobi) hat inn belehrt, „dass wahre Schätzung nicht ohne Schonung sein kann." Vielleicht haben auch die Schlegel beigetragen. Im Oktober 1799 waren sie viel mit Goethe in Jena. Mit Friedrich nahm Goethe seine Gedichte durch. Das „Athenäum“

1) Medicus, Eiṇleitung, p. CV. 
Sie bilden die grossen Tendenzen des Jahrhunderts. ${ }^{1}$ ) Goethe erkundigt sich bei Friedrich nach Fichtes Wohlergehen. Fichte grollt. „Es ist begreiflich, dass Leute wie Goethe, nachdem nur die erste Hitze bei ihnen verraucht ist, sich des Auftritts mit mir, der ihnen, wie sie wohl wissen, auch noch ganz anders gedeutet wird, als sie meinen, schämen, ihn ungeschehen wünschen. Es ist leicht, in der Hitze einen falschen Schritt durchzusetzen, aber sehr schwer, ihn bei kaltem Blute wieder gut zu machen." So Fichte an Johanna (20. Okt. 1799).

Später kommen Nachrichten aus Berlin. Fichte besteigt die Rostra der Weltgeschichte, neben der das Jenenser Katheder wie ein Spielzeug verschwindet. Der spekulierende Wissenschaftslehrer von Jena wird zum Staatsphilosophen und Pädagogen, der Jacobiner von ehedem znm moralischen Überwinder Napoleons, zum glühenden Patrioten und philosophischen Begründer des deutschen Einheitsgedankens, der "Atheist" von einst zum religiösen Heilslehrer lässt unter allen lebenden Deutschen nur Fichte und Goethe gelten. und Menschheitsführer. Der alternde Goethe verfolgt seinen Weg. "Mit wahrer Liebe und Bewunderung" spricht er von Fichtes Antwortschreiben an Reinhold. Das Dokument habe inn ergriffen und stellenweise erschüttert. ${ }^{2}$ ) Von Fichtes didaktischer Tätigkeit kommt inm 1805 manches Gute $\mathrm{zu}^{{ }^{3}}$ ) Allmählich verwischt die Zeit alte Gegensätze. "Im Sommer 1810 begegnen sich beide Männer in Teplitz und ihr Zwist ist begraben. Goethe sieht den Redner an die deutsche Nation die Promenade hinunterschreiten und spricht zu Zelter: „Da geht der Mann, dem wir alles verdanken!“

Ein Stück Weltgeschichte geht an Deutschland vorüber. Schiller, Fichte sinken vor dem Allesüberlebenden dahin. Der Tod verklärt das Gewesene. "Glückliche Zeit, heisst es 1817, da der treffliche Fichte, dieser kräftige und entschiedene Mann, noch persönlich unter uns lebte und wirkte! "4) Und 1820 schliesst der Greis in den "Tag- und Jahresheften" die Akten über den vor ihm Dahingeschwundenen. „Er war eine der tüchtigsten Persönlichkeiten, die man je gesehen, und an seinen Gesinnungen in höherem Betracht nichts auszusetzen."

1) Johanna an Fichte, Leben I, 324, 325.

2) Schelling an Fichte am 24. Mai 1801. Leben II, $504 \mathrm{ff}$.

of An J. v. Muiller, 25. Jan. 1805.

4) Schriften zur Literatur. Jub.-Ausg. Bd. 37, S. 96. 
Für Fichtes Weiterentwicklung war die Jenenser Katastrophe von grösster Bedeutung. Und dies in doppeltem Sinn. Der Philosoph hat sein Zeitalter von der schlimmsten Seite kennen gelernt. Fichte verkannt! Welche Erfahrùng! Seine Verachtung der Menschen, seine Missstimmung namentlich gegen die wissenschaftliche Welt wächst ins Ungeheure. „Was hinter dem Rücken von mir geredet wird, das höre ich nicht. Dies ist die einzige Weise, um durch eine solche Lumpenwelt zu kommen" (an Johanna, 28. Okt. 1799). Im "Anti-Nicolai", in den "Grundzügen des gegenwärtigen Zeitalters" hat sich Fichte seinen Groll von der Seele geredet. Mit der Verachtung der gegenwärtigen wächst aber zugleich auch die Sehnsucht nach einer zukünftigen besseren Menschheit and das Verlangen, an ihrer Bildung mitzuarbeiten. In der „Bestimmung des.Menschen " klingt diese neuerwachte $\mathrm{Kultursehnsucht}$ zum ersten Male an. Sie bleibt von nun an der Sinn all seines Strebens.

Und dann: das religiöse Problem hat sich inm geklärt und dies wiederum im engsten Anschluss an persönlichste Jenenser Erfahrungen. Im Herbst 1799 zurückgezogen in Berlin lebend, dem Drang der Geschäfte, dem enggeistigen Kleinstadtleben enthoben, betrachtet er von einer höheren Warte die menschlichen Dinge. Wie lächerlich gering erscheinen sie da! Err erhebt sich zum Absoluten, zur Spinozistischen "species aeternitatis", und jeder niedrige Groll schwindet aus seinem grossen Herzen. „Ich habe bei der Ausarbeitung meiner gegen wärtigen Schrift (der „Bestimmung des Menschen") einen tieferen Blick in die Religion getan als noch je. Bei mir geht die Bewegung des Herzens nur aus vollkommener Klarheit hervor; es konnte nicht fehlen, dass die errungene Klarheit zugleich mein Herz ergriff" (an Johanna, 5. Nov. 1799). Und weiter: "Glaube mir, dass diese Stimmung an meiner unerschütterlichen Freudigkeit und an der Milde, womit ich die Ungerechtigkeit meiner Gegner ansehe, grossen Anteil hat. Ich glaube nicht, dass ich obne diesen "fatalen Streit und ohne die bösen Folgen desselben jemals zu dieser klaren Einsicht und zu dieser Herzensstimmung gekommen wäre."

Und das Urteil der Geschichte? Zwei wahrhaft grosse Menschen, deren Seelen sich suchten und deren Lebenswege eng verflochten waren. Und die sich doch nicht vereinigen konnten nach dem schmerzlich-resignierten Geständnis Schillers, weil die hobe Weisheit, dass wahre Schätzung nicht obne Schonung sein kann, so leicht im Lärm des Lebens verhallt. 